\title{
Comparative Evaluation of Two Established Age Estimation Techniques (Two Histological and Radiological) by Image Analysis Software using Single Tooth
}

\author{
Madhu Shrestha* \\ Chitwan Medical College, Oral Pathology, Bharatpur-10, Nepal
}

\begin{abstract}
There are a variety of techniques for accurate estimation of age from teeth that offer a reasonable degree of accuracy, but it still remains a dilemma for the inexperienced practitioner to standardize a particular one. For this reason this study intends to standardize an age estimation method for the Indian population. Age estimation in this study was done by two established radiological methods like (Tooth Coronal index (TCl) and Kvaal's method) and two histological techniques (Kashyaps and Koteswara modification of Gustafson's method and secondary dentine method). A total of forty-one extracted teeth of varied age and gender, of known age, sex and teeth number were taken, photographed and numbered for blind fold study. A new technique of radiographic method using a modified paralleling technique with grid lines was employed. Radiological parameters such as "crown height", "pulp height" and were measured for TCl whereas "height" and "width" at three different points were taken for Kvaal's technique. Morphological parameters such as attrition, histological parameters such as secondary dentin, root transparency and cementum apposition were assessed from ground sections. TCI method was found to be the best radiologic and secondary dentin estimation was found to be the best histological techniques.
\end{abstract}

Keywords: Forensic science; Age estimation; Gustafson's method; Tooth coronal index; Kvaal's method; Image analysis

\section{Introduction}

Identification of a person is the first basis for any forensic or medico-legal process. It is an important basis for differentiating guilty from innocent in legal issues, for ethical issues and for declaration of death reports and the basis for probing into criminal cases, mass disaster or war victims. The accurate estimation of age at the time of death with the help of dental remains is an important parameter for identification. Human tooth can be preserved for a long time after death without gross changes due to which it serves as an important tool in forensic science. Adult teeth consist of enamel as the outermost covering of tooth crown and dentin underneath, both of which are hard tissues resistant to decomposition, followed by pulp as the innermost soft tissue core. Likewise cementum is the outermost covering for the surface of root which is also resistant to decomposition.

With the first introduction of usage of teeth for estimation of age by Gustafson [1], various methods of age estimation have been described and modified. Age estimation in children and adolescents can be performed with a standard technique given by Schour and Massler's [2], Demirijian's method [3]; Schour and Massler's chart and also the developing third molar method [2]. When we talk of age estimation in adults' dentition, Gustafson's method [1] is still in practice by the forensic odontologists in medicolegal issues, although a lot number of modifications to this method have been described. Kashyap and Koteswara [4] modified Gustafson's criteria to make it more objective and feasible in skeletal remains. Root translucency $[5,6]$ and incremental lines in cementum [7] have also proved to be promising methods. However both these methods require tooth extraction followed by sectioning/processing that may not be possible to estimate age in living individuals. Kvaal et al. [8] introduced a radiographic method in which tooth extraction was not required as it was done by indirectly measuring deposition of secondary dentin (by measuring pulp radiolucency) and correlated it to age. This study followed the method of Ikeda et al. [9] as thus tooth-coronal index (TCI) after was computed for each tooth and regressed on real age. Previous research by Ito et al. and Ikeda et al. [911] has estimated age through anatomical crown and the constriction of the coronal pulp cavity using radiographs of histological sections of adult teeth.

Many of these studies have discussed methods based on measurements on various radiographic and morphological parameters and developed formulas on such basis [12]. However, the reproducibility of these parameters is uncertain, as the values may be different for individuals from different ethnical groups. The present study has used morphological and radiological parameters and supplemented both with image analysis technique to calculate age and have derived a formula that can serve as a reference for further large scale studies.

\section{Materials and Methods}

Forty-one extracted permanent teeth, having varied age and gender distribution and known age, sex and tooth number were taken. The teeth were numbered to avoid the biasness and to cover the identity preventing bias. The age group was divided into four groups from thirteen to sixty-five years. Teeth that were fractured, restored, and teeth with gross evidence of hyper-cementosis or grossly decayed were excluded. The gross and physical attributes such as color, measurements and other changes were noted and photographs taken. The gender and age distribution of the patients are shown in Table 1.

*Corresponding author: Madhu Shrestha, Chitwan Medical College, Oral Pathology, Bharatpur-10, Nepal, Tel: 977-9851136008; E-mail: drmadhushrestha@gmail.com

Received May 19, 2014; Accepted July 29, 2014; Published August 01, 2014

Citation: Shrestha M (2014) Comparative Evaluation of Two Established Age Estimation Techniques (Two Histological and Radiological) by Image Analysis Software using Single Tooth. J Forensic Res 5: 237 doi:10.4172/21577145.1000237

Copyright: (c) 2014 Shrestha M. This is an open-access article distributed under the terms of the Creative Commons Attribution License, which permits unrestricted use, distribution, and reproduction in any medium, provided the original author and source are credited. 
Citation: Shrestha M (2014) Comparative Evaluation of Two Established Age Estimation Techniques (Two Histological and Radiological) by Image Analysis Software using Single Tooth. J Forensic Res 5: 237 doi:10.4172/2157-7145.1000237

Page 2 of 6

\begin{tabular}{|l|c|c|c|c|}
\hline Age group & Age in years & Number & Male & Female \\
\hline Young & $0-20$ & 12 & 10 & 2 \\
\hline Adolescents \& Adults & $21-40$ & 5 & 4 & 1 \\
\hline Adults & $41-60$ & 12 & 10 & 2 \\
\hline Older & $>60$ & 12 & 6 & 6 \\
\hline
\end{tabular}

Table 1: The gender and age distribution of the patients.
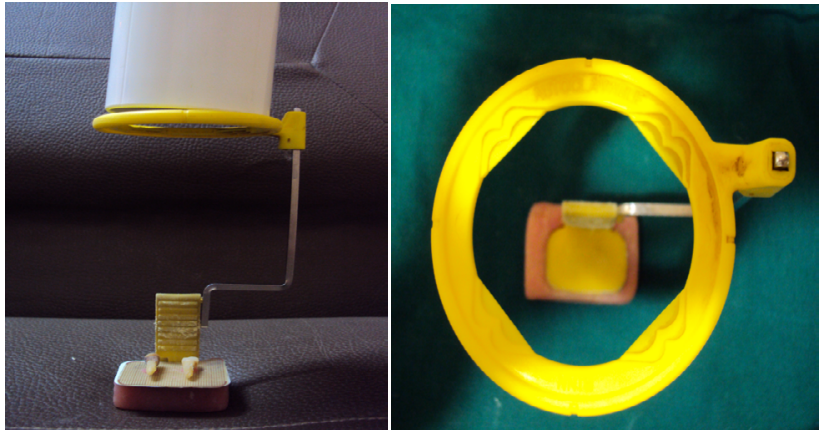

Figure 1: A and B: IOPA radiographs of teeth using a customized acrylic base with modified paralleling cone technique and gridlines (appendix 1).

The total sample of forty-one teeth was divided into four groups. First group named as "young" included age group from zero to twenty years, twenty to forty years as second group (adolescents and adults), forty to sixty as group three (adults) and above sixty as group four (older) respectively. Age estimation was carried out with two histological and two radiological methods. The parameters used for radiographic methods were crown height $(\mathrm{CH})$, coronal pulp cavity height $(\mathrm{CPCH})$, and secondary dentin deposition by measuring pulp radiolucency. In the Kvaal's radiographic method [8] different parameters like tooth length, root length, pulp length were taken. Similarly, root width and pulp width at three different points like at cemento- enamel junction (CEJ), at halfway of root and midway between the given two points were taken.

The histological parameters used from ground sections of teeth were measurements of incisor edge width, measurement at cervical margins, translucency of root, and secondary dentin deposition. The histological parameters were carried with macroscopic and microscopic examination.

The radiographic method implemented was modified to the usual conventional technique. The impression for the base of the plastic ring was made with alginate impression and the cast was used to prepare the acrylic stand for the base of the plastic ring. This acrylic stand was fitted onto the base of the plastic ring, the film (KODAK IOPA) was secured on top of it. Then a radiographic grid was placed on top and teeth were radiographed with their long axis perpendicular to the film with paralleling technique. An IOPA (intraoral periapical) radiograph of each tooth was taken mesio-distally with a paralleling cone technique that was secured with an acrylic film holder especially designed for this study. A radiopaque gridline was used for accurately checking the dimensions (Figures 1 and 2). Then morphological radiological parameters were assessed on the radiographs using Image Analysis Software (Image J version 7). Then teeth were sectioned into half with a rotating disc and ground sections were made. Assessment of histological parameters was carried out as described in detail in the following headings. All the values were calculated separately using Image analysis software to prevent errors by manual measurement.
The estimated age calculated by these various methods were compared with the known real age of the individual. Statistical analysis was done.

\section{Estimation of Tooth Coronal Index (TCI) [9]}

The technique described by Ikeda et al. [9] was slightly modified as Image analysis software was used on the photographs of the radiographs. Parameters such as crown height $(\mathrm{CH})$ [9] and coronal pulp cavity height $(\mathrm{CPCH})[9]$ were calculated. For this purpose, a straight line was traced between the cemento-enamel junction of the tooth which is the division between the anatomical crown and root. The crown height $(\mathrm{CH})$ was measured vertically from the cervical line to the tip of the highest cusp [9]. The coronal pulp cavity height (CPCH) was measured vertically from the cervical line to the tip of the highest pulp horn [9]. Teeth with marked attrition were excluded. The following formula was calculated.

Tooth-coronal index (TCI) for each tooth: $\mathrm{TCI}=\mathrm{CPCH} \times 100 / \mathrm{CH}$ [9] (Figure 3).

\section{Estimation of Secondary Dentine Deposition on Radiographs (by measuring pulp radiolucency) [8]}

Secondary dentin deposition was carried by measuring pulp radiolucency on radiographs as described by Kvaal et al. [8] with slight modifications. In this study Image analysis software was implemented on the photographs of the radiographs to calculate various measurements on the tooth and pulp. Parameters measured were tooth length, the pulp length and root length on the mesial surface from the cemento-enamel junction (CEJ) to the root apex [8]. The root

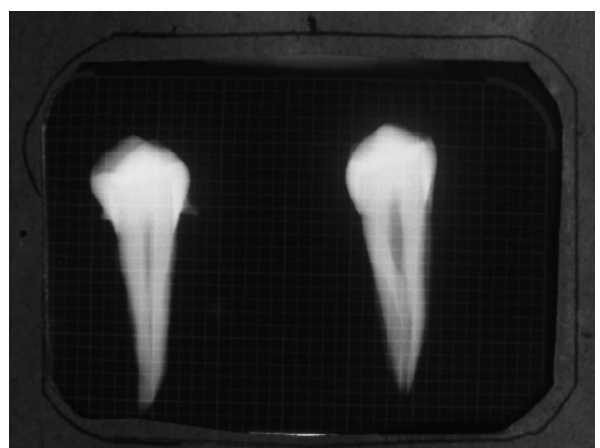

Figure 2: IOPA radiographs obtained with modified paralleling cone technique (appendix 1).

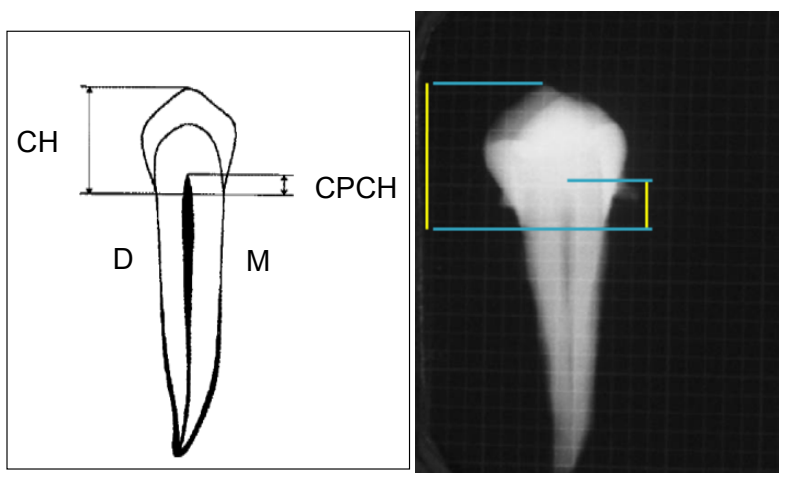

Figure 3: Tooth coronal index method [9] implemented on radiographs with gridlines using Image analysis (appendix 2). 
and pulp width both at the CEJ (level A) and at the midroot level, i.e. halfway between the CEJ and the apex of the root (level C), as well as at the midpoint between the CEJ and mid-root level (level B) [8] were measured with image analysis (Figure 4).

\section{Measurement of Dentine Translucency [13]}

Secondary dentin was calculated using image analysis of the photographs of ground sections of teeth against a calibrated scale. Dentin translucency was measured as the distance between the apical limit and the most coronal extent of translucency within the root. An appropriate light source and contrast background was used while measuring [13] (Figure 5).

\section{Koteswara and Kashyap's modification of Gustafson's method [4]}

Four variables as given by Koteswara and Kashyap [4] such as attrition, secondary dentin deposition, cementum deposition at the root apex, dentin translucency were used to assess the age from the ground sections of teeth using image analysis. Both microscopic and macroscopic measurements were taken for the following parameters. Calculations were carried out for attrition index, premolar and molar attrition index values, secondary dentin deposition, translucency

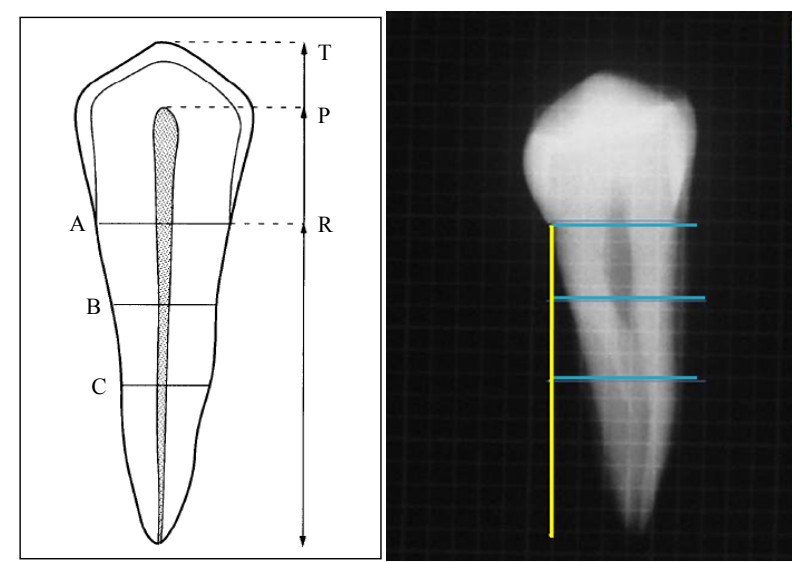

Figure 4: Kvaal's method [8] of secondary dentin deposition (by measuring coronal pulp radiolucency) implemented on radiographs with gridlines using Image analysis (appendix 3).

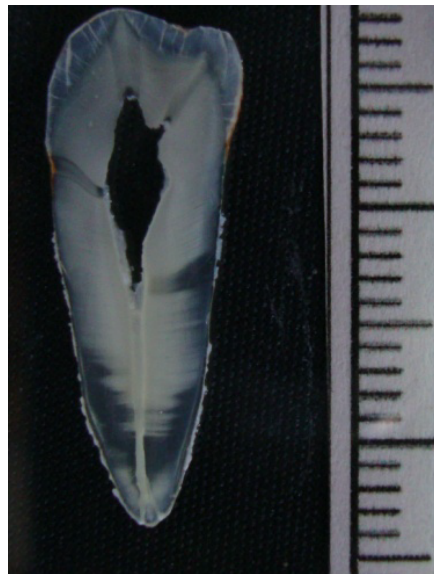

Figure 5: Measurement of dentin translucency by Image analysis (appendix 4).

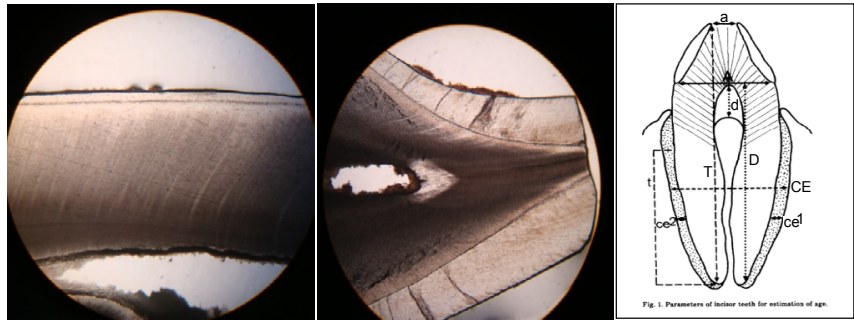

Figure 6: Measurement of secondary dentin formation and cementum thickness [4] by Image analysis (appendix 5).

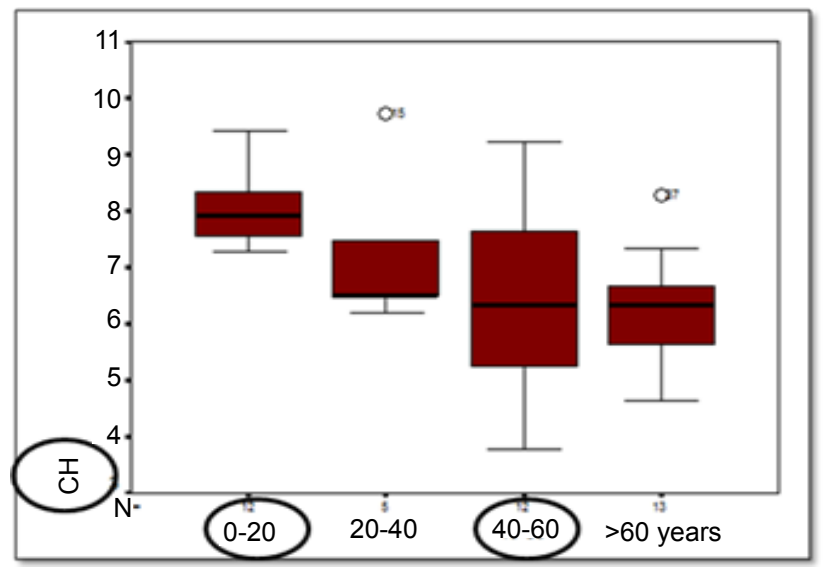

Figure 7: Bar diagram of crown height.

of the root, and cementum apposition using same formulae as given by Koteswara and Kashyap [4] (Figure 6).

\section{Statistical analysis}

Statistical analyses were carried out using SPSS version 11.5. KruskalWallis test was done for the histological and morphological parameters. It was followed by post-hoc Bon- ferronis correction.

\section{Results}

The study had forty one teeth, out of which thirty were of male and eleven were of female. These were divided into four groups, young group ranged ( $0-20$ years) comprised of twelve sample, second group adolescents and adults (21-40 years) had a total number of five, adults group (41-60 years) and the last older age group ( $>60$ years) had a total sample of twelve. According to the radiological parameters in the study, in TCI method (originally given by Ikeda et al.) [9], Kruskall-Wallis test showed that both crown height and tooth coronal index as given by ( $\mathrm{TCI}=\mathrm{CPCH} \times 100 / \mathrm{CH}$ ) were found to be significant parameters. The crown height $(\mathrm{CH})$ was significantly decreased with increase in with a $p$ value of $(0.003)$. TCI is also significantly increased with increase in age with a $\mathrm{p}$ value of (0.009). According to the Post-hoc Bonferroni's correction when TCI was compared amongst the various age groups, it was significant to differentiate between young $(0-20)$ age group and middle age group of (40-60) years and between older teeth ( $>60$ years) with a p value of (0.011) and (0.00) 7 respectively. However, TCI is a more useful clinical parameter as it helps to differentiate between the young (0-20) and adolescents and adults (21-40) groups significantly with a p value of (0.002) (Figures 7-11).

In the Kvaals method [8] only the pulp width at the CEJ parameter 


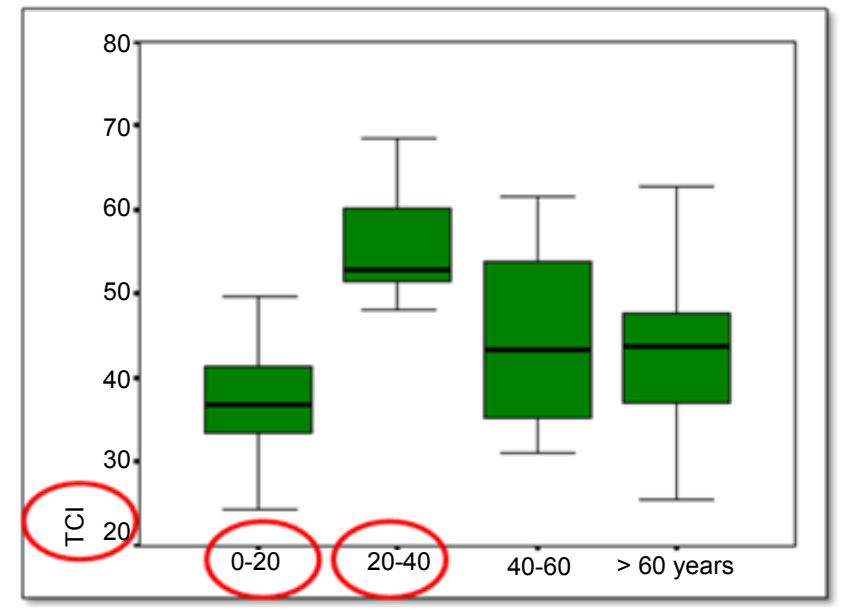

Figure 8: Bar diagram of TCl.

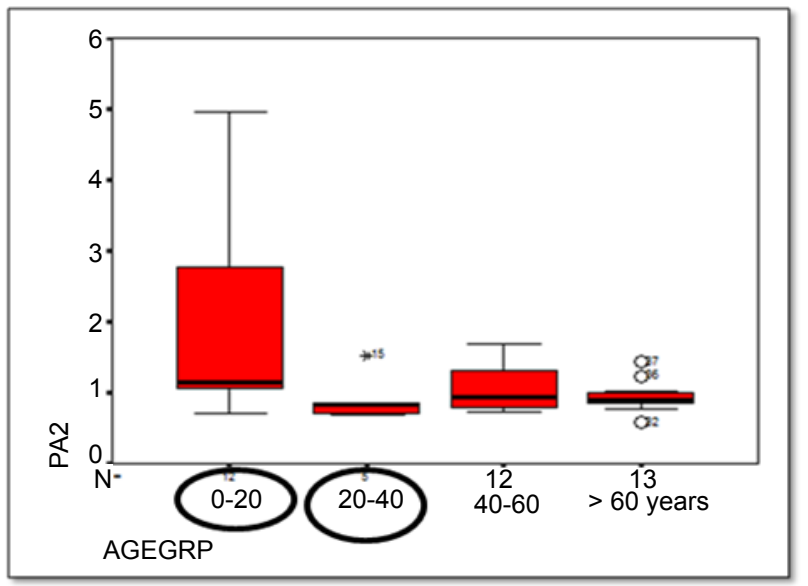

Figure 9: Bar diagram of pulp width at CEJ (given as PA2) of Kvaal's Method.

(given as PA2 in table) was found to be significant with a $\mathrm{p}$ value of (0.023) by Kruskall-Wallis test. When Post-hoc Bonferroni's correction was done it was found to be significant in differentiating age between young $(0-20)$ and older age groups ( $>60$ years) with a $p$ value of $(0.007)$.

Among the various histological parameters, dentin translucency was found to be increasing significantly with increase in age with a 'p' value of (0.006) by Kruskall-Wallis test in our study. Post hoc Bonferroni's correction showed that it was also helpful to differentiate between teeth of young ( 0 -20 years) and adults (41-60 years) age group with a ' $p$ ' value of $(0.002)$ and between young $(0-20)$ and old $(>60)$ with a 'p' value of (0.004).

Out of the four parameters of Kashyap's and Koteswara, only dentin translucency and secondary dentin (both microscopic and macroscopic) indices were found to be significant in estimating age by Kruskall -Wallis test. Translucency index of Kashyap's and Koteswara [4] was significantly increased with increase in age with a 'p' value of (0.004). Post hoc Bonferroni correction showed that it was useful to differentiate between age groups of young (0-20), adults (41-60) and older (>60 years) with 'p' value of (0.002) and (0.004) respectively.

Secondary dentin index was found to be significant with a 'p' value of (0.032) macroscopically and (0.003) microscopically. Post hoc
Bonferroni's correction showed that both macroscopic and microscopic values were significant in differentiating between age groups of (0-20), $(40-60)$ and $>60$ years with ' $p$ ' values of $(>0.05)$. Forward stepwise linear regression was done to identify a established formula by combination of the significant parameters.

\section{Regression analysis for radiological and histologic parameters}

Histologic Predictive Formulas H (Estimated Age)=-12.709+0.774 (T I)+5.067 (CAI; macroscopic) Radiographic Predictive Formulas $\mathrm{H}$ $($ Estimated Age $)=54.422-1.3498(\mathrm{CH})+3.444(\mathrm{PA} 2)+8.47(\mathrm{CPCH})$

\section{Discussion}

Edwin Saunders [14] showed that teeth were more accurate assessment of age than height. Gustafson [1] made the earliest systematic attempt to estimate age from radiographs, using macro structural change and is the most popular age estimation test employed by forensic odontologists and pathologists. Since then age estimation using dental parameters has been used in forensic science [14]. Kashyap modified Gustafson's criteria by omitting gingival recession and root resorption from the original work. This study also followed the same

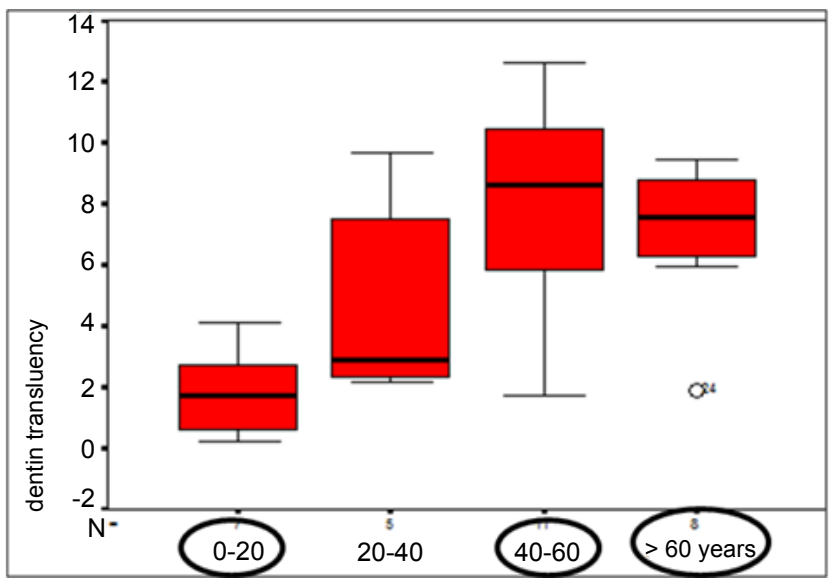

Figure 10: Bar diagram of dentin translucency.

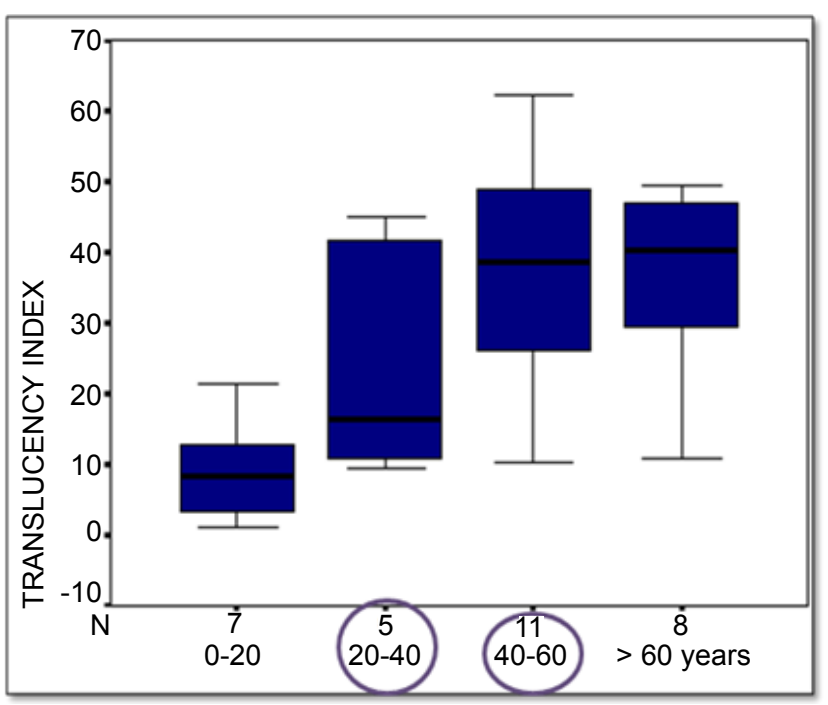

Figure 11: Bar diagram of translucency index. 
method as the histological technique but a linear regression was done for calculating the formula.

Paewinsky et al. [15] did a study on OPGs from 168 individuals to estimate age by Kvaal's method. According to their study [15], the best correlations were at level A (pulp width at CEJ) with the exception of mandibular first premolars where the best correlation was at level B (i.e at midpoint between the CEJ and mid-root level B) The same level was found to be significant in our study, however, we did not check each individual tooth for best results.

Nathalie Bosmans et al. [16] had used Kvaal's original technique on orthopantomographs but it was aided by the computer program Adobe Photoshop 6.01 (Adobe Systems $\backslash$ Incorporated, San Jose, CA, USA) to measure the maximum tooth length, the pulp length, the root length on the mesial surface from the ECJ to the root apex, the root and pulp width at levels A (pulp width at CEJ), B (i.e at midpoint between the CEJ and mid-root level), and C (i.e. halfway between the CEJ and the apex of the root). No significant intra-observer effects were found between measurements by the two techniques and the calculated age did come very near to the chronological age.

Kullman et al. [17] had used Kvaal's method on radiographs both with image analysis and manual measurements.

Kolltveit and Solheim et al. [18] had conducted a study to compare the reliability of manual measurements (vernier calipers using a stereomicroscope) with that of computer-assisted measurements (CAM) of morphological parameters using Image analysis software in dental radiographs. Ratios calculated from the linear measurements of "tooth" by "pulp" showed a weaker correlation with age when the image analysis program was employed than did ratios based on conventional measurements (without image analysis) of the same. The main source of errors in measurement seemed to be difficulties in recognition of the reference points on the radiographs when viewed on the monitor, and therefore in defining the line to be measured.

All the radiographs taken in our study were first modified to correctly mark the three reference points by a straight line. This image was then saved in JPEG file and opened with Image Analysis software. Thus, we tried to minimize the errors identified in previous studies. The best correlation in our study was also made from the pulp width at CEJ; i.e. at level A. This correlation was useful in estimating between the (0$20)$ and ( $>60$ years) age. But this point cannot be applied clinically as it was significant to differentiate age groups between the two extremes (such as young and older). Other studies have shown that calculations of radiographic pulp has a stronger correlation with chronological age than measured length of a tooth; thus is valuable as a biomarker for generalized ageing [19].

Drusini et al. [11] had, partially followed the "TCI" technique described Ikeda et al. [9] using intact teeth instead of histological sections. The measurements were taken directly from the radiographs. According to their study, the length of the coronal pulp cavity $(\mathrm{CPCH})$ showed a significant correlation with chronological age.

When "TCI" [9] was determined in our study using "CPCH" [9] and " $\mathrm{CH}$ " [9], crown height $(\mathrm{CH})$ and tooth coronal index (TCI=CPCH $\mathrm{X} 100 / \mathrm{CH}$ ) were found to be significant parameters whereas $\mathrm{CPCH}$ alone was insignificant in estimating age unlike the findings by Drusini et al. [11]. However, "TCI" [9] is more useful clinically as it helps to differentiate between the (0-20 years) and (20-40 years) groups significantly with a $\mathrm{p}$ value of $(0.002)$.
"Secondary dentin" is the calcified nontubular substance deposited by the pulp on the walls of the pulp chamber and root canal and it continues throughout life $[11,13]$. Since regular secondary dentin is laid down on the pulpal surface of the primary dentin, the pulp cavity decreases in size with age [19].

Bodecker identified that the apposition of secondary dentin strongly correlated to chronological age. With advancing age, the intratubular calcification of dentin increases, Difference between intra and extra tubular inorganic material is equalized resulting in the appearance of "dentinal translucency" at the apical region of tooth of old age. Dentin translucency can be correctly used to estimate age as given by Johanson and Bang et al. [13]. According to our study the "dentin translucency" parameter is highly effective in determining the correct age of the individual. With increase in age there was an increase in translucency of dentin. In addition, it was also useful clinically as it helps to differentiate between the teeth of young and old age group. Only translucency and secondary dentin (both microscopic and macroscopic) indices were found to be significant in estimating age by Kruskall-Wallis test. The present study showed that secondary dentin formation is an efficient parameter as it is deposited along the wall leading to a reduction in the size of the pulp cavity.

Similarly other studies have shown that age has a greater influence on secondary dentin formation than does dental wear or irritation [19].

The histologic method showed that the age could be calculated using the translucency index and cementum apposition index (macroscopic reading). Similarly in the radiological estimation it was seen that crown height and coronal pulp cavity height along with TCI method was the best in calculating the age in the Indian population. In the given study the use of image analysis procedure was followed to ensure the reduction in bias that could have occurred with the observer's make it more accurate than the manual method.

\section{Conclusion}

Accurate estimation of age is not only important for identification of the deceased victim but also in medico legal issues as well as biological anthropology. As we have various techniques of age estimation, it is practically very difficult for an inexperienced practitioner to decide a particular one. This study was performed to standardize a technique after comparison among various techniques. Although histologic technique is accurate, it is more time-consuming and is a destructive approach and is important for postmortem cases as any teeth may be used but less practical for living individual where tooth has to be extracted. In contrast, radiographic techniques are less time consuming with no need of extraction, can be applied to both living and dead and there is no use of specialized equipment. Among all the histological parameters, the best was the translucency index which was applicable both clinically and the histological Predictive formula derived from the given study was:

\section{$[\mathrm{H}($ Estimated Age $)=-12.709+0.774(\mathrm{TI})+5.067(\mathrm{CAI} ;$ macroscopic $)]$}

When we compare the various radiological methods, TCI was the most reliable biomarker for age estimation which is even useful clinically.

The Radiographic Predictive Formulas was:

$\mathrm{H} \quad($ Estimated Age $)=54.422-1.3498 \quad(\mathrm{CH})+3.444 \quad(\mathrm{PA} 2)+8.47$ $(\mathrm{CPCH})$

Amongst all techniques, the present study highlights the importance 
Citation: Shrestha M (2014) Comparative Evaluation of Two Established Age Estimation Techniques (Two Histological and Radiological) by Image Analysis Software using Single Tooth. J Forensic Res 5: 237 doi:10.4172/2157-7145.1000237

Page 6 of 6

of secondary dentin formation to accurately estimate the age which can be deduced by TCI. Thus, it was concluded that Tooth Coronal Index known as TCI can be used as a reliable parameter for age estimation using single tooth in forensic investigation of individuals of unknown data and dental anthropology. It is best of use when Image $J$ and radiographic gridlines are incorporated.

\section{References}

1. Gustafson G (1950) Age determination on teeth. J Am Dent Assoc 41: 45-54.

2. Schour I, Massler M (1940) Studies in tooth development the growth pattern of human teeth. J Am Dent Assoc 27:1918-1931.

3. Demirjian A, Goldstein H, Tanner JM (1973) A new system of dental age assessment. Hum Biol 45: 211-227.

4. Kashyap VK, Koteswara Rao NR (1990) A modified Gustafson method of age estimation from Teeth. Forensic Sci Int 47: 237-247.

5. Bang G (1989) Age changes in teeth: Developmental and regressive. In MY Isc, an (ed.): Age Markers in the Human Skeleton. Springfield: Thomas CC $211-235$

6. Bang G, Ramm E (1970) Determination of age in humans from root dentin transparency. Acta Odontol Scand 28: 3-35.

7. Kagerer P, Grupe G (2001) Age-at-death diagnosis and determination of lifehistory parameters by incremental lines in human dental cementum as an identification aid. Forensic Sci Int 118: 75-82.

8. Kvaal SI, Kolltveit KM, Thomsen IO, Solheima Tore (1995) Age estimation of adults from dental radiographs. Forensic Sci Int 74: 175-185.
9. Ikeda N, Umetsu K, Kashimura S, Suzuki T, Oumi M (1985) Estimation of age from teeth with their soft X-ray findings. Nihon Hoigaku Zasshi 39: 244-250.

10. Ito S (1972) Research on age estimation based on teeth. Jpn J Legal Med 26: 31-41.

11. Drusini AG, Toso O, Ranzato C (1997) The Coronal Pulp Cavity Index: A Biomarker for Age Determination in Human Adults. Am J Phys Anthropol 103: 353-363.

12. Kvaal S, Solheim T (1994) A non-destructive method for age estimation. Forensic Odontostomatol 12: 6-11.

13. Solheim T (1989) Dental root translucency as an indicator of age. European Journal of Oral Sciences 97: 187-197.

14. Ajmal M, Mody B, Gopa Kumar (2001) Age Estimation using Three established methods. Forensic Science International 122: 150-154.

15. Paewinsky E, Pfeiffer H, Brinkmann B (2005) Quantification of secondary dentine formation from orthopantomograms-a contribution to forensic age estimation methods in adults. Int J Legal Med 119: 27-30.

16. Bosmansa N, Annb P, Alya M, Willems G (2005) The application of Kvaal's dental age calculation technique on panoramic dental radiographs. Forensic Science International 153: 208-212.

17. Kullman L, Tronje G, Teivens A, Lundholm A (1996) Methods of reducing observer variation in age estimation from panoramic radiographs. Dentomaxillofac Radiol 25: 173-178.

18. Kolltveit KM, Solheim T, Kvaal SI (1998) Methods of measuring morphological parameters in dental radiographs. Comparison between image analysis and manual measurements. Forensic Science International 94: 87-95.

19. Babshet M, Acharya AB, Naikmasur VG (2010) Age estimation in Indians from pulp/tooth area ratio of mandibular canines. Forensic Sci Int 197: 125e1-125e4. 